\title{
Network Video Transmission Quality Assurance Algorithm Based On Congestion Control
}

\author{
Honggao Zhu \\ School of Information and Technology \\ HubeiVocational and Technical college \\ Xiaogan,Hubei, China \\ e-mail: hbzyzhu@163.com
}

\author{
Qi Feng \\ Business School \\ Newcastle University, Newcastle Upon Tyne \\ United Kingdom \\ springbell_feng@qq.com
}

\begin{abstract}
Time delay and packet loss rate during the network video transmission are the two important factors in network video transmission quality. The purpose of congestion control is to reduce the bad impact of network video transmission quality caused by time delay and packet loss rate. This paper proposed a network video transmission quality assurance algorithm based on unbalanced multiple descriptions coding scheme. The new algorithm predicts the congestion state of network by means of Markov model based on available bandwidth detection, changes video transmission path on the basis of the prediction. Experiments show that compared with RED (Random Early Detection) algorithm, the algorithm is much more effective to estimate network congestion、 reduce video packet loss rate and time delay, thus the network video transmission quality can be ensured more effectively.
\end{abstract}

Keywords-Unbalanced Multiple Description Coding ; Bandwidth Detection; Markov Model; Optimal Route Selection

\section{INTRODUCTION}

As the Internet and multimedia technology development, the network video transmission of real-time has become one of the important applications. However, network traffic congestion phenomenon such as high time delay and packet loss rate causes great influence to video transmission quality. In order to improve the quality of network video transmission, the domestic and foreign experts and scholars carried out many research and put forward many video coding scheme and congestion control algorithm. Multiple Description Coding (MDC) encodes video into independent description of adapting to different network bandwidth, so that it can to establish contact with the most conform network bandwidth. Generally speaking, most of the MDC use several independent video descriptions for transmission ${ }^{[1-2]}$. In order to recover the relevant video quality, the receiver decoders need one or more description. Unbalance Multiple Description Coding (UMDC) [2] can control flexibly redundant arising from encoding by video description of the multi-different and makes full use of available bandwidth of different paths in the network. RED (Random Early Detection) algorithm ${ }^{[3]}$ that was proposed by S. Floyd etc. is the most typical of AQM (Active Queue Management) congestion control algorithm, and has now become only AQM candidate strategy in the IETF (Internet Engineering Task Force). Although RED algorithm can avoid global synchronous phenomenon and effectively improve the bandwidth utilization rate and reduce average queue length, it has also many problems need to be solved, such as, in a given flow condition that steady-state error of the packet queue length 、 delay jitter, in different flow condition that a lot of packet loss caused by the change greatly of average queue length unable effectively to estimate the seriousness of the congestion etc. In order to solve these problems to ensure video transmission of realtime and decoding quality in the receiving end, UMDC is adopted in this paper and the algorithm which can effectively reduce the time delay and packet loss rate is put forward based on the effective predicts the state of network congestion to improve video transmission efficiency. Simulation results show that, compared with the RED algorithm, the new algorithm can effectively predicts the state of network congestion, reduce the lost package rate and time delay, thus it can more effectively ensure the network video transmission quality.

\section{UnBalance Multiple Description Coding} (UMDC)

According to the importance of each description and divide weight of description, Multiple Description Coding can be divided into Balanced Multiple Description Coding (BMDC) and UMDC which uses video description of several different weight and quality. We adopted two variables High-Resolution (HR) that is used to describe higher weight and quality and Low-Resolution (LR) that are used to hide HR and to describe packet loss errors in the transmission process as redundant [6-7].

This paper introduces the concept of "synchronous frame”, which is I-th frame of LR-encoder reconstruction. In order to replace the current $P$ frame reconstructed by HRencoder in the frame buffer of HR-encoder, we adopt the relevance both LR-encoder input and reconstruction frame of HR-encoder to instead of the original video frame, at the same time, the next $\mathrm{P}$ frame encoded in HR-encoder is used as the reference frame. In this paper, we use "synchronous frame" to strengthen the correlation between HR and LR description in the end of encoder, meanwhile, HR-decoder must obtain "synchronous frame" for the next $\mathrm{P}$ frame motion compensation in the end of decoder.

In this paper, by using "synchronous frame" to enhance the correlation of HR description and LR description, we can deal with a variety of packet loss situation. The ways of working how to enhance the correlation of HR description 
and LR description between encoder and decoder are to be discussed in the following three points. (1) when HR description and LR description go well received, the two describe can correct decoding and the date of HR description is outputted in the decoder, or decoder just begin to decode after waiting for the next "synchronous frame" arrive, and the decoder outputs the decoded frames obtained in the last time during the suspension of decoding process; (2) when HR description received smoothly and LR lost in the transmission, LR-encoder inputs the reconstruction frame of HR-encoder, at this time need only is that the output of HR-decoder inputs directly to a standard encoder, in order to generate LR description to correct reconstruct the damaged frames for packet loss and continue to decoder of LR description; (3) when LR description received smoothly and HR lost in the transmission, as the "synchronous frame" is the reference frame of next $\mathrm{P}$ frame of HR description, so do not match station is not exist between HR-encoder and HR-decoder, at the time need only is to get the next "synchronous frame" from the output of LR-decoder so that HR-decoder can start again decoding.

\section{NETWORK DATA TRANSMISSION ROUTE SELECTION AND IMPLEMENTATION ALGORITHM}

\section{A.One-way delay detection}

Network transmission delay is one of the important parameters to measure the network performance, which can be divided into one-way delay and two-way delay. As for data packet may experience different path in coming and going transmission process, and UMDC concerns mainly the one-way path from sender to recipient, so one-way transmission delay is more meaningful to network video. One-way network transmission delay of measuring procedure is as follows:

The first step: to negotiate clock synchronization both the sender and the receiver.

The second step: test data packets are generated in the sender according to TCP (Transmission Control Protocol) structure, in which the receiver IP (Internet Protocol) address is filled and the timestamp is set, and of which using random bits is to fill the vacancy part, then the test date packets filled out are send to the destination host.

The third step: to calculate one-way transmission delay after the corresponding test data packets are received in the receiving end.

\section{B.Available bandwidth detection}

Define parameter $R D_{d}=\left(R D_{a v g}-R D_{\min }\right) /\left(R D_{\max }-\right.$ $R D_{\text {min }}$ ), among that $R D_{\text {avg }}, R D_{\min }, R D_{\max }$ are respectively average transmission delay, minimum transmission delay and maximum transmission of $n$ test date packets. When the sending rate of test date packets is greater than available bandwidth, it causes the growth of test date flow delay, by definition it also presents the growth trend; otherwise the test data flow delay will not change trend, or by definition it will be close to zero. The experiment shows that, if the parameter value is set to 0.45 , the error in both the detected available bandwidth and the actual available bandwidth approximate to 0 .

Define parameter $R D_{n}=R D_{\text {avg }} / R D_{\text {max }}-R D_{\text {min }} / R D_{\text {avg. }}$. By comparison with the range ability of the received test packet sending delay, we can adjust the test packet sending rate so that the detection rate is fast approaching to the available bandwidth. When the test packet sending rate is less than the current available network bandwidth, the values of $R D_{\text {avg }}, R D_{\max }$ and $R D_{\min }$ close, and the $R D_{n}$ value is close to 0 ; otherwise, the $R D_{n}$ value presents a growing trend. The experiment shows that, if the $R D_{n}$ value set to 0.017, the error in both the detected available bandwidth and the actual available bandwidth approximate to 0 .

The algorithm of the available bandwidth detection can be described as follows:

The first step: initialization $S_{\max }$ is the physical bandwidth of the bottleneck link, $S_{\min }$ and $S_{\text {now }}$ is zero, among them, $S_{\max }, S_{\min }$ and $S_{\text {now }}$ are respectively maximum, minimum, and real-time values of probe packets sending rate;

The second step: setting $S_{\text {now }}=\left(S_{\max }+S_{\text {min }}\right) / 2$, to send the detect packets;

The third step: calculate $R D_{d}$ and $R D_{n}$ when the destination end receives 100 detect packets.

The forth step: when $R D_{d}>0.45$ and $R D_{n}<0.017$, the output link bandwidth detection value is $S_{\text {now }}$, then the available bandwidth detection end.

The fifth step: when $R D_{d}>0.45, S_{\max }=S_{\text {now }}$; or else $S_{\min }$ $=S_{\text {now }}$ and $S_{\text {now }}$ is recalculated according to equation (1);

$S_{\text {now }}=S_{\text {max }} *\left(1-R D_{\mathrm{d}}^{2}\right)+S_{\min } * R D_{\mathrm{d}}^{2}$

The sixth step: go to the second step.

\section{C.Network congestion prediction}

In the certain time interval, the network congestion status has the statistical regularity of the discrete Markov chain. Using the Markov model to predict network congestion status is to estimate the network congestion state transition probability matrix, the general use of statistical estimation method to estimate the network state transition probability matrix ${ }^{[8]}$.

Definition of network congestion state space $\mathrm{S}=$ \{idle, normal, congestion , in two consecutive periods suppose that the probability is $P_{i j}(\mathrm{i}=1,2, \ldots, \mathrm{n})$ in condition that the network congestion is $i$ state in the previous time and $j$ state in the latter time, among $P_{i j}$ said the possibility of state i into state $\mathrm{j}$, and then construct the step transition probability matrix $P$.

Set the vector $X(\mathrm{t})=\left(X_{1}(t), X_{2}(t), \ldots, X_{n}(t)\right)$ is the distribution vector of the $\mathrm{t}$-th period of network congestion status, (2) can be calculated in the $(t+1)$-th period of network congestion status, and thus to predict the next network status.

$\left[\begin{array}{c}X_{1}(\mathrm{t}+1) \\ X_{2}(\mathrm{t}+1) \\ \ldots \\ \mathrm{X}_{\mathrm{n}}(\mathrm{t}+1)\end{array}\right]=\left[\begin{array}{c}X_{1}(\mathrm{t}) \\ X_{2}(\mathrm{t}) \\ \cdots \\ \mathrm{X}_{\mathrm{n}}(\mathrm{t})\end{array}\right] P=\left[\begin{array}{c}X_{1}(\mathrm{t}) \\ X_{2}(\mathrm{t}) \\ \cdots \\ \mathrm{X}_{\mathrm{n}}(\mathrm{t})\end{array}\right]\left[\begin{array}{ccc}P_{11} & P_{12} & P_{13} \\ P_{21} & P_{22} & P_{23} \\ P_{31} & P_{32} & P_{33}\end{array}\right]$ 


\section{D.Transmission path selection}

Definition of $F_{l}=\frac{\mathrm{r}_{l}}{C_{l}}+\frac{\mathrm{C}_{l}}{C_{l}-\mathrm{r}_{l}}$ for the link transmission capacity factor, which is used to reflect the data transmission capability of the next hop link, among them $C_{l}$ is the physical bandwidth of link $l, \Gamma_{l}$ is the available bandwidth of link $l . F_{l}$ is the greater, and data transmission capacity of the link is the stronger, which will be selected as the greater the probability of the transmission path. Assume that the next hop has $\mathrm{n}$ paths, to calculate $\mathrm{n}$ paths $F_{l}$, the corresponding paths of all $F_{l}$ maximum are selected as the transmission paths, if the corresponding paths are not unique, it will be selected randomly a path as the transmission path [9].

\section{E. Implementation algorithm}

In the proposed algorithm, using UMDC for video codec, network one-way delay is measured to detect the available bandwidth of the current video transmission path. On this basis, we can use of Markov model to predict network status and select the transmission path through the link transmission capacity factor $\mathrm{Fl}$, in which the sender can adjust the video sent bit rate.

\section{Sender algorithm description}

Step 1: to establish a TCP connection with the receiving end;

Step 2: video encoding and set the initial send bit rate;

Step 3: to send the test packet and calculate the available bandwidth of the each transmission path based on the feedback of the receiving end and select a maximum link of the $F_{l}$ as the video transmission path;

Step 4: according to equation (2), to predict network link congestion status and adjust dynamically the video sent bit rate. If the video transmission path is idle or normal state, it needs to increase the video sent bit rate; then to lower the video sent bit rate in the congestion state, otherwise turn to Step 3 ;

Step 5: to send the video description;

Step 6: if receive the stop video communication instructions, it needs to send the packets containing the stop video communication signs for the receiving end, otherwise turn to Step 3.

\section{Receiving end algorithm description}

Step 1: to establish a TCP connection with the sender;

Step 2: to receive detection data packets and make a statistic to the packets delay;

Step 3: to calculate parameters $R D_{d}$ and $R D_{n}$, carry out bandwidth detection algorithm, and make the feedback of algorithm execution results to the sender;

Step 4: to receive video description and use UMDC method for video decoding;
Step 5: if receive the packet containing the stop video communication signs, the bandwidth detection algorithm and proposed algorithm are terminated, otherwise turn to Step 2.

\section{EXPERIMENTAL RESULTS AND ANALYSIS}

The experiment simulation platform is Open-net. The transmission video streams from a typical video test sequences foreman use the TCP burst flow to generate congestion, to select the 180 seconds experimental fragment from simulation experiment. The simulation results are as follows:
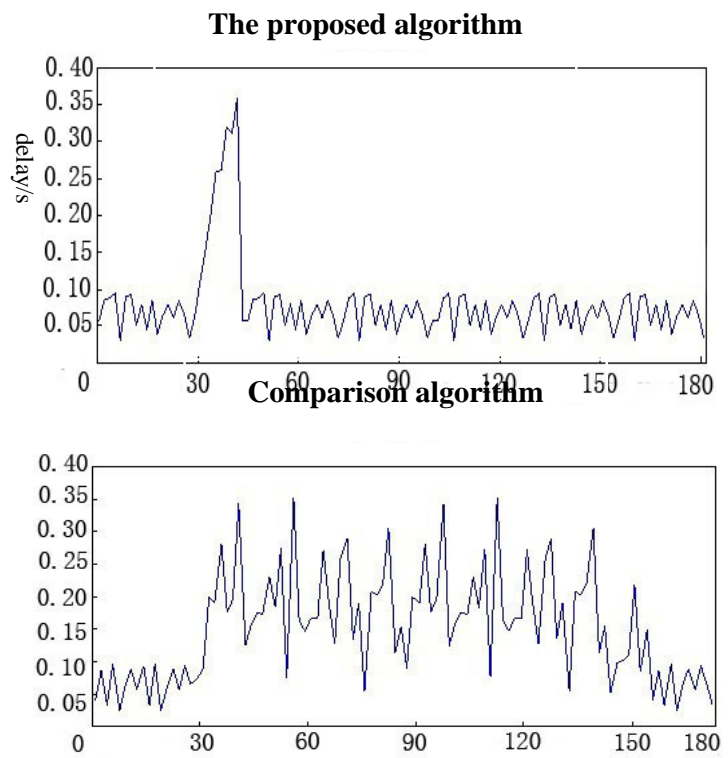

Fig. 1 Compare the delay of the proposed algorithm with the RED algorithm.

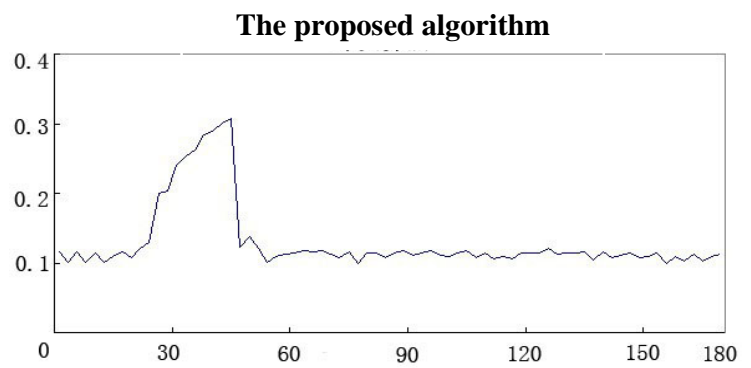

Comparison algorithm 


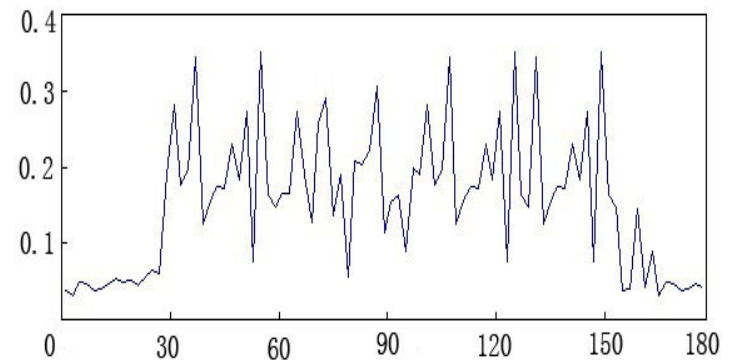

Fig. 2 Compare the packet loss rate of the proposed algorithm with the RED algorithm.

From Fig. 1 and Fig. 2, we can see that, after using the algorithm to predict congestion, the certain amount of time is consumed for optimal route selection. In the proposed algorithm, the delay and packet loss rate are increasing steadily, which are greater than the RED algorithm at the same period, but with the assurance of optimal transmission path, the delay and packet loss rate decrease rapidly, which are less than the RED algorithm at the same period. The average delay and packet loss rate of the proposed algorithm and the RED algorithm are calculated from the simulation data, which are respectively $0.1086 \mathrm{~s}$ and $0.1309 \mathrm{~s} 、 0.1963 \mathrm{~s}$ and $0.1987 \mathrm{~s}$. From the whole point of view, this paper algorithm is more effective than the RED algorithm by controlling congestion to ensure the quality of video transmission.

\section{CONCLUSIONS}

Network congestion has a serious impact on the network video transmission quality, a network video transmission quality assurance algorithm is proposed in this paper, in which we adopt UMDC to code video, to predict network congestion based on the real-time available bandwidth description using Markov model, to replace of transmission path for the optimal path. The simulation results show that, in the network congestion, the network video transmission quality assurance algorithm proposed is more effective than the RED to estimate the congestion status, to reduce the network delay and packet loss rate, to ensure the video transmission quality.

\section{REFERENCES}

[1] Vaishampayan V. A., Design of multiple description scalar quantizers, IEEE Transaction on Information Theory, 1993, 39: 821-834

[2] Apostolopoulos J. G. and Wee S. J., Unbalanced Multiple Description Video Communication Using Path Diversity, Proceedings of IEEE International Conference on Image Processing (ICIP'01), Thessaloniki, Greece, 2001: 966-969.

[3] Floyd S ,Jacobson V .Random early detection gateways for congestion avoidance. IEEE /ACM Transaction on Networking. August 1993 ,1(4): 397-413

[4] RYU S, RUMP C, QIAO C. Advances in Internet congestion control. IEEE Communications Surveys \& Tutorials, Third Quarter,2003, 5(1) : 28-39.

[5] Wang Hua-bin. RED Algorithm Research and Simulation Analysis Based On NS2. [J] Computer Systems \& Applications. 2008, 17 (12).

[6] Apostolopoulos J. G. and Wee S. J., Unbalanced Multiple Description Video Communication Using Path Diversity, Proceedings of IEEE International Conference on Image Processing (ICIP’01), Thessaloniki, Greece, 2001: 966-969.

[7] Li Bin, Huang Feng, Sun Li-Feng, Yang Shi-Qiang. A Robust and Flexible Unbalanced Multiple Description Video Coding and Transmission Scheme. Journal of Computers. [J] 2008, 31(7).

[8] Shi Hong-Tao. Periodic Traffic Prediction Algorithm of Markov Based on Normal Smooth Sequence. Journal of Qingdao Agricultural University(Natural Science). 2010, 27 (4).

[9] Guan Li-An, Wang Bin-Qiang, Zhu Xuan-Yong. Path Selection Algorithm Based on Residual Bandwidth and Link Utilization Rate of Next Hop. Telecommunications Science. 2010, 26 (8). 